Pacific Journal of Mathematics

ON COMMUTATIVE, NONPOTENT ARCHIMEDEAN 


\title{
ON COMMUTATIVE, NONPOTENT ARCHIMEDEAN SEMIGROUPS
}

\author{
RICHARD G. LEVIN
}

In this paper we will study commutative, archimedean, nonpotent (i.e., without an idempotent) semigroups, obtaining several results concerning finitely generated ones. The main theorem of this paper is the following: a finitely generated, commutative, archimedean, nonpotent semigroup is power joined. The main theorem is derived by considering the decomposition of the semigroup $S$ into a union of disjoint semilattices; the congruence $\rho_{b}$, defined by $x \rho_{b} y$ if and only if there exist positive integers $n$ and $m$ such that $b^{n} x=b^{m} y$, determines the union, whereas congruence classes are semilattices under the partial order $\geqq_{b}$ defined by $x \geqq_{b} y$ if and only if $y=b^{n} x$ or $y=x$. The set of maximal elements relative to $\geqq_{b}$ generates $S$. The following is a crucial lemma in the proof of the main theorem: let $S$ be a finitely generated, commutative, nonpotent, archimedean semigroup; then the set of maximal elements of $S$ relative to $\geqq_{b}$ is a finite set.

Let $S$ be a commutative, nonpotent, archimedean semigroup. We will define a congruence $\rho$ on $S$ and state several results concerning $S / \rho$ and the congruence classes of $S$ modulo $\rho$. The remarks and definitions which precede Definition 5 will be used in several instances; a complete discussion can be found in [5]. See [6] and [7] for an abstract of these results. Proofs of all other results in this paper are supplied.

Definition 1. Let $b \in S$. The binary relation $\rho_{b}$ on $S$ is defined by $x \rho_{b} y$ if and only if there exist positive integers $n$ and $m$ such that $b^{n} x=b^{m} y$.

The relation $\rho_{b}$ is a congruence relation on $S$ and $b$ is called the standard element determining the corresponding decomposition of $S$. Furthermore, for any $b, S / \rho_{b}$ is a group; the congruence class modulo $\rho_{b}$ containing $b$ is the identity element of $S / \rho_{b}$ and it is a subsemigroup of $S$. We call $S / \rho_{b}$ the structure group of $S$ with respect to $b$.

Definition 2. Let $S_{\alpha}$ be an arbitrary congruence class of $S\left(\bmod \rho_{b}\right)$. The following relation, $\geqq_{b}$ is a partial order on $S_{\alpha}$. Let $x, y \in S_{\alpha}$. We define $\geqq_{b}$ on $S_{\alpha}$ by $x \geqq_{b} y$ if and only if there exists a positive integer $n$ such that $y=b^{n} x$, or $y=x$.

Definition 3. A discrete tree $R$ is a lower semilattice (i.e., a 
partially ordered set in which every pair of elements have a greatest lower bound) satisfying:

(a) for all $x, y, z \in R, x<z$ and $y<z$ imply $x \leqq y$ or $y \leqq x$, and

(b) the set $\{x \mid x \in R$ and $b \leqq x \leqq c\}$ is a finite set for any pair $b, c \in R$.

Let $S_{\alpha}$ be a congruence class of $S$ modulo $\rho_{b}$. Then $S_{\alpha}$ is a discrete tree with respect to the partial order $\geqq_{b}$.

DEFinition 4. An element $x$ of $S$ is called a prime element of $S$ relative to the congruence $\rho_{b}$ if $x$ is not divisible by $b$. Or, alternately, $x$ is a prime element if $x$ is a maximal element of a congruence class $S_{\alpha}$ of $S\left(\bmod \rho_{b}\right)$ relative to the partial order $\geqq_{b}$ defined on $S_{\alpha}$.

The following two remarks are particularly useful.

REMARK 1. Let $a \in S$. Then

$$
\bigcap_{n=1}^{\infty} a^{n} S=\varnothing
$$

REMARK 2. Let $a, b \in S$. Then

$$
a \neq a b \text {. }
$$

DEFINITION 5. Let $R$ be an arbitrary semigroup. We define the binary relation $\leqq$ on $R$ by $a \leqq b$ if and only if there exists $x \in R$ such that $a=b x$, or $a=b$. If $a \neq b$ and $a \leqq b$ we generally write $a<b$.

LEMMa 1. Let $S$ be a finitely generated, commutative, nonpotent, archimedean semigroup. Then the relation $\leqq$ on $S$ is a partial order and $S$ satisfies the ascending chain condition relative to $\leqq$.

Proof. It follows from the definition that reflexivity is satisfied. Suppose that $a, b \in S$ and $a \leqq b$ and $b \leqq a$. Then either $a=b x$ and $b=a y$, or $a=b$. Consider the former. We conclude that

$$
a=(a y) x=a(y x) \text {. }
$$

But (1) contradicts Remark 2. Therefore $a=b$. Thus, $\leqq$ is antisymmetric. Suppose $a \leqq b$ and $b \leqq c$. We suppose also that $a \neq b$ and $b \neq c$. Then $a=b x$ and $b=c y$ for some $x, y \in S$. Therefore,

$$
a=(c y) x=c(y x) .
$$

Thus, $a \leqq c$, and now it is obvious that $\leqq$ is transitive.

Suppose there exists a sequence of elements of $S,\left\{a_{n} \mid n>0\right\}$, 
satisfying

$$
a_{1}<a_{2}<a_{3}<\cdots<a_{n} \cdots \text {. }
$$

Let $T=\left\{\alpha_{1}, \alpha_{2}, \cdots, \alpha_{n}\right\}$ be a finite generating set of $S$. The sequence (3) reduces to the set of equations:

$$
a_{n}=a_{n+1} x_{n+1}, \quad \text { for all } n \geqq 1 \text {, where } x_{n+1} \in S \text {. }
$$

The set of equations (4) leads to the set

$$
a_{1}=a_{2} x_{2}=a_{3} x_{2} x_{3}=\cdots=a_{n} x_{2} x_{3} \cdots x_{n}=\cdots
$$

For all $k, x_{k}$ can be expressed as

$$
x_{k}=\alpha_{1}^{r_{k 1}} \alpha_{2}^{r_{k 2}} \cdots \alpha^{r_{k n}},
$$

and $r_{k j} \geqq 0$ for $j=1,2, \cdots, n$, and there exists $j_{0}, 1 \leqq j_{0} \leqq n$ such that $r_{k j 0}>0$. Multiplying the $x_{i}$ 's, we arrive at

$$
\begin{aligned}
& x_{2}=\alpha_{1}^{r_{21}} \alpha_{2}^{r_{22}} \cdots \alpha_{n}^{r_{2 n}} \\
& x_{2} x_{3}=\alpha_{1}^{p_{31}} \alpha_{2}^{p_{32}} \cdots \alpha_{n}^{p_{3 n}} \\
& x_{2} x_{3} \cdots x_{k}=\alpha_{1}^{p_{k 1}} \alpha_{2}^{p_{k 2}} \cdots \alpha_{n}^{p_{k n}},
\end{aligned}
$$

where $0 \leqq r_{2 j} \leqq p_{3 j} \leqq p_{4 j} \leqq \cdots \leqq p_{k j} \leqq \cdots$, for $j$ satisfying $1 \leqq j \leqq n$. If for some $j, 1 \leqq j \leqq n$, we have

$$
\lim _{n \rightarrow \infty} p_{n j}=+\infty \text {, }
$$

then we can write

$$
a_{1}=\alpha_{j} y_{1}=\left(\alpha_{j}\right)^{2} y_{2}=\left(\alpha_{j}\right)^{3} y_{3}=\cdots=\left(\alpha_{j}\right)^{k} y_{k}=\cdots,
$$

and we conclude that

$$
a_{1} \in \bigcap_{n=1}^{\infty}\left(\alpha_{j}\right)^{n} S
$$

This contradicts Remark 1 . We set

$$
R_{j}=\lim _{n \rightarrow \infty} P_{n j}
$$

and

$$
M=\max \left\{R_{1}, R_{2}, \cdots, R_{n}\right\} .
$$

The number $M$ is finite and it is now obvious that there exists an integer $N \geqq M$ such that

$$
x_{2} x_{3} \cdots x_{N}=x_{2} x_{3} \cdots x_{N} x_{N+1} \text {. }
$$

This contradicts Remark 2, and the contradiction establishes that $S$ 
satisfies the ascending chain condition relative to the relation $\leqq$.

LEMMA 2. Let $S$ be a commutative, nonpotent, archimedean semigroup and let $\leqq$ be the partial order on $S$ defined above (see Definition 4 ). Let $S$ satisfy the ascending chain condition relative to $\leqq$. Then the set of maximal elements relative to $\leqq$ is a generating set for $S$ and is contained in every other generating set of $S$.

Proof. Let $S^{\prime}$ be an arbitrary generating set for $S$ and let $S^{\prime \prime}$ be the set of all the maximal elements of $S$ relative to $\leqq$. Let $\beta \in S^{\prime \prime}$. Then

$$
\beta=\alpha_{1} \alpha_{2} \cdots \alpha_{n}, \text { where } \alpha_{i} \in S^{\prime}
$$

Suppose $n>1$. Then $\beta=\alpha_{1}\left(\alpha_{2} \cdots \alpha_{n}\right)$. This implies that $\beta<\alpha_{1}$. Since this is impossible, $n=1$ and $\beta \in S^{\prime}$. That is, $S^{\prime \prime} \leqq S^{\prime}$.

Let $x \in S$. Then

$$
x=\alpha_{1} \alpha_{2} \cdots \alpha_{n}, \quad \text { where } \alpha_{i} \in S^{\prime} \text { for } 1 \leqq i \leqq n .
$$

Fix $t, 1 \leqq t \leqq n$. Suppose $\alpha_{t}$ is not a maximal element. Then there exists $\beta_{t} \in S^{\prime \prime}$ such that $\alpha_{t}<\beta_{t}$ and $\alpha_{t}=\beta_{t} x_{t}$ for some $x_{t} \in S$. If $x_{t}$ is not a maximal element then there exists a maximal element $\beta_{t 1}$ such that $x_{t}=\beta_{t 1} x_{t 1}$. By definition of $\leqq, x_{t}<x_{t 1}$. We continue in this fashion. After $N$ steps we arrive at the equation

$$
\alpha_{t}=\beta_{t} \beta_{t 1} \beta_{t 2} \cdots \beta_{t, N-1} x_{t, N-1}
$$

and the sequence of inequalities

$$
\alpha_{t}<x_{t}<x_{t 1}<x_{t 2} \cdots<x_{t, N-1},
$$

where $\beta_{t, k}$ is a maximal element for $1 \leqq k \leqq N-1$. Since $S$ satisfies the ascending chain condition, we conclude that this procedure must lead to a maximal element $x_{t, M}$ in (14) and (15). Setting $M=N-1$ in (14) and substituting (14) into (13) we express $x$ as a product of maximal elements. We conclude that $S^{\prime \prime}$ generates $S$ and that $S^{\prime \prime}$ is the smallest generating set of $S$.

Proposition 3. Let $S$ be a finitely generated, commutative, nonpotent, archimedean semigroup. Then the set of maximal elements (relative to $\leqq$ ) of $S$ is a finite set.

Proof. According to Lemma 1, $S$ satisfies the ascending chain condition relative to $\leqq$. By Lemma 2, the set of maximal elements of $S$ is a subset of every generating set of $S$. Since $S$ is finitely generated, the set of maximal elements of $S$ must be a finite set. 
Proposition 4. Let $S$ be a finitely generated, commutative, nonpotent, archimedean semigroup. Let $a \in S$. Then the set of prime elements of $S$, with respect to the standard element $a$, is a finite set.

Proof. Suppose there are an infinite number of primes. Let $S^{\prime}=\left\{b_{1}, b_{2}, \cdots, b_{k}, \cdots\right\}$ be a countably infinite subset of the set of primes of $S$. Let $T=\left\{a_{1}, a_{2}, \cdots, a_{n}\right\}$ be the set of all maximal elements of $S$. Every element of $S^{\prime}$ admits a representation of the form

$$
b_{j}=a_{1}^{\mu_{j 1}} a_{2}^{\mu}{ }_{j 2} \cdots a_{i}^{\mu_{j i}} \cdots a_{n}^{\mu_{j n}}, \mu_{j i} \geqq 0 .
$$

Consider the sequences

$$
\mu_{1 i}, \mu_{2 i}, \mu_{3 i}, \cdots, \mu_{k i}, \cdots, \text { where } 1 \leqq i \leqq n .
$$

For at least one $i$ between 1 and $n$ the corresponding sequence (17) will be unbounded. Otherwise we immediately conclude that $S^{\prime \prime}$ is a finite set. Suppose the sequence for $i_{0}$ is unbounded. Choose a subsequence

$$
\mu_{t_{1}, i_{0}}, \mu_{t_{2}, i_{0}}, \cdots, \mu_{t_{r}, i_{0}}, \cdots
$$

satisfying

$$
\mu_{t_{1}, i_{0}}<\mu_{t_{2}, i_{0}}<\cdots .
$$

For convenience, we will change the notation. Set

$$
r_{j}=\mu_{t_{j}, i_{0}}, \quad \text { for } j \geqq 1 \text {. }
$$

We now have

$$
b_{j_{k}}=a_{1}^{(1} \cdots a_{i_{0}}^{r_{k}} \cdots a_{n}^{()}, \quad \text { for } k \geqq 1 .
$$

For all $k, b_{j k} \neq a x_{k}$ for any $x_{k} \in S$. But since $S$ is an archimedean semigroup, there exists an integer $l, l>0$ such that

$$
\left(a_{i_{0}}\right)^{l}=a \mu \text {. }
$$

There exists $k_{0}>0$ such that $r_{k}>l$ for all $k \geqq k_{0}$. Therefore we have

$$
\begin{aligned}
b_{j_{k}} & =a_{1}^{(1} \cdots a_{i_{0}}^{r_{k}} \cdots a_{n}^{(1)} \\
& =a_{1}^{(1)} \cdots a_{i_{0}}^{(} a_{i_{0}}^{r_{k}-l} \cdots a_{n}^{(1)} \\
& =a \mu\left(a_{1}^{()} \cdots a_{i_{0}}^{r_{k-l}} \cdots a_{n}^{(1)}\right), \quad \text { for all } k \geqq k_{0}
\end{aligned}
$$

This contradiction establishes that the set of primes is a finite set.

Proposition 5. Let $S$ be a finitely generated, commutative, nonpotent, archimedean semigroup. Let $\rho_{b}$ be the congruence relation of Definition 1. Then $S / \rho_{b}$ is a finite group. 
Proof. The elements of $S / \rho_{b}$ are congruence classes. Each congruence class is a tree and contains at least one prime element. If $S / \rho_{b}$ were an infinite group, then $S$ would contain an infinite number of prime elements. This would contradict Proposition 4. Thus $S / \rho_{b}$ is a finite group.

THEOREm 1. A finitely generated, commutative, nonpotent, archimedean semigroup is power joined.

Proof. Let $a \in S$. Consider $S / \rho_{a}$. Let $x, y \in S$. Let $\alpha, \beta \in S / \rho_{a}$ such that $x \in S_{\alpha}$ and $y \in S_{\beta}$. Since $S / \rho_{a}$ is a finite group, there exist positive integers $n$ and $m$ such that $\alpha^{n}=\varepsilon, \beta^{m}=\varepsilon$, where $\varepsilon$ is the identity of $S / \rho_{a}$. Therefore, $x^{n} \in S_{\varepsilon}$ and $y^{m} \in S_{\varepsilon}$. Let $\left\{P_{1}, P_{2}, \cdots, P_{r}\right\}$ be the set of prime elements of $S$ which are contained in $S_{\varepsilon}$. Let

$$
P=g l b\left\{P_{1}, P_{2}, \cdots, P_{r}\right\},
$$

where the partial order in $S_{\varepsilon}$ is $\geqq_{a}$ (see Definition 1). Set

$$
T=\left\{Z \mid Z \in S_{\varepsilon}, Z \geqq{ }_{a} P\right\} \text {. }
$$

$T$ is a finite set because $S_{\varepsilon}$ is a discrete tree. Since $S$ is nonpotent, the powers of $x^{n}$ and $y^{m}$ are all distinct. Therefore there exist positive integers $r, t$ such that $P \geqq_{a}\left(x^{n}\right)^{r}$ and $P \geqq_{a}\left(y^{m}\right)^{t}$. Further, for all $Z$, where $P \geqq_{a} Z$, there exists a positive integer $s$ such that $Z=a^{s}$. Therefore,

$$
\left(x^{n}\right)^{r}=a^{\mu_{1}},\left(y^{m}\right)^{t}=a^{v_{1}},
$$

and

$$
\left(x^{n r}\right)^{v_{1}}=\left(y^{m t}\right)^{\mu_{1}}
$$

We conclude that $S$ is a power joined semigroup.

THEOREM 2. Let $S$ be a commutative, nonpotent, archimedean semigroup. Let $a \in S$ and let $G_{a}\left(=S / \rho_{a}\right)$ be the corresponding structure group. Then, $S$ is power joined if and only if $G_{a}$ is a periodic group and $S_{\varepsilon}$ is power joined (where $S_{\varepsilon}$ is the congruence class of $S \bmod \rho_{a}$ which contains a).

Proof. Let $S$ be power joined. Let $\alpha \in G_{a}, y \in S_{\alpha}$. There exist positive integers $n$ and $m$ such that $y^{n}=a^{m}$. Since $a^{m} \in S_{\varepsilon}$, so is $y^{n}$. Therefore $\alpha^{n}=\varepsilon$ and we conclude that $G_{a}$ is periodic. The set $S_{\varepsilon}$ is power joined because it is a subsemigroup of $S$.

To prove the converse, let $x, y \in S$. There exist $\alpha, \beta \in G_{a}$ such that $x \in S_{\alpha}, y \in S_{\beta}$ and $\alpha^{n}=\varepsilon, \beta^{m}=\varepsilon$. Therefore, $x^{n} \in S_{\varepsilon}, y^{m} \in S_{\varepsilon}$. Since 
$S_{\varepsilon}$ is power joined, there exist positive integers $k$ and $l$ such that

$$
\left(x^{n}\right)^{k}=\left(y^{m}\right)^{l} \text {. }
$$

We conclude that $S$ is power joined.

THEOREM 3. Let $S$ be a commutative, nonpotent, archimedean semigroup. Then $S$ is power joined if and only if every finitely generated subsemigroup is archimedean.

Proof. Let $S$ be power joined. Let $S^{\prime}$ be a finitely generated subsemigroup of $S$. Then $S^{\prime}$ is also power joined. Let $x, y \in S^{\prime}$. Then there exist positive integers $n, m$ such that $x^{n}=y^{m}$. Set $\mu=$ $y^{m-1}, v=x^{n-1}$. We get $x^{n}=y \mu$ and $y^{m}=x v$. The elements $\mu$ and $v$ are also in $S^{\prime}$. In case $n$ or $m$ equals 1 , we can easily arrange the desired equations by multiplying both sides of the equation $x^{n}=y^{m}$ by $x$ or $y$ as required. Therefore $S^{\prime}$ is archimedean.

Let $x, y \in S$. Let $S^{\prime}$ be the subsemigroup of $S$ generated by $x$ and $y$. Since $S^{\prime}$ is finitely generated, it is archimedean. Thus, $S^{\prime}$ is a finitely generated, commutative, nonpotent, archimedean semigroup, and by Theorem 1 we conclude that $S^{\prime}$ is power joined. Therefore, there exist positive integers $n$ and $m$ such that $x^{n}=y^{m}$. Since $x$ and $y$ were arbitrary elements of $S$, we now conclude that $S$ is power joined.

\section{REFERENCES}

1. James Christlock, The structure of archimedean semigroups, Thesis, University of California, Davis, 1966.

2. A. H. Clifford and G. B. Preston, The algebraic theory of semigroups, Volume 1, Amer. Math. Soc., Providence, R. I., 1961.

3. Mario Petrich, On the structure of a class of commutative semigroups, Czechoslovak Math. J. (89), 14 (1964) Prague.

4. Takayuki Tamura, Commutative, nonpotent, archimedean semigroups with cancellation law 1, J. of Gakugei, Tokushima Univ. 8 (1957), 5-11.

5. Construction of commutative, archimedean semigroups, (to be published in Math. Nachr., Germany)

6. - Notes on commutative, archimedean semigroups I, Proc. Japan Acad. 42 (1966), 35-40.

7. - Notes on commutative, archimedean semigroup II, Proc. Japan Acad. 42 (1966), 545-548.

Received November 7, 1967. This paper contains part of a doctoral dissertation written under the direction of Professor Takayuki Tamura.

The University of California, Davis, and

Western Washington State College, Bellingham 



\section{PACIFIC JOURNAL OF MATHEMATICS}

\section{EDITORS}

\section{H. ROYDEN}

Stanford University

Stanford, California

\author{
R. R. Phelps \\ University of Washington \\ Seattle, Washington 98105
}

\section{J. DugundJI}

Department of Mathematics University of Southern California Los Angeles, California 90007

\section{RICHARD ARENS}

University of California

Los Angeles, California 90024

\section{ASSOCIATE EDITORS}

\section{E. F. BECKENBACH}

B. H. NEUMANN

F. WOLF

K. YOSIDA

\section{SUPPORTING INSTITUTIONS}

UNIVERSITY OF BRITISH COLUMBIA CALIFORNIA INSTITUTE OF TECHNOLOGY UNIVERSITY OF CALIFORNIA MONTANA STATE UNIVERSITY UNIVERSITY OF NEVADA NEW MEXICO STATE UNIVERSITY OREGON STATE UNIVERSITY UNIVERSITY OF OREGON OSAKA UNIVERSITY UNIVERSITY OF SOUTHERN CALIFORNIA

\author{
STANFORD UNIVERSITY \\ UNIVERSITY OF TOKYO \\ UNIVERSITY OF UTAH \\ WASHINGTON STATE UNIVERSITY \\ UNIVERSITY OF WASHINGTON \\ AMERICAN MATHEMATICAL SOCIETY \\ CHEVRON RESEARCH CORPORATION \\ TRW SYSTEMS
}

NAVAL WEAPONS CENTER

Mathematical papers intended for publication in the Pacific Journal of Mathematics should be in typed form or offset-reproduced, double spaced with large margins. Underline Greek letters in red, German in green, and script in blue. The first paragraph or two must be capable of being used separately as a synopsis of the entire paper. It should not contain references to the bibliography. Manuscripts, in duplicate if possible, may be sent to any one of the four editors. All other communications to the editors should be addressed to the managing editor, Richard Arens, University of California, Los Angeles, California 90024.

Each author of each article receives 50 reprints free of charge; additional copies may be obtained at cost in multiples of 50 .

The Pacific Journal of Mathematics is published monthly. Effective with Volume 16 the price per volume (3 numbers) is $\$ 8.00$; single issues, $\$ 3.00$. Special price for current issues to individual faculty members of supporting institutions and to individual members of the American Mathematical Society: $\$ 4.00$ per volume; single issues $\$ 1.50$. Back numbers are available.

Subscriptions, orders for back numbers, and changes of address should be sent to Pacific Journal of Mathematics, 103 Highland Boulevard, Berkeley 8, California.

Printed at Kokusai Bunken Insatsusha (International Academic Printing Co., Ltd.), 7-17, Fujimi 2-chome, Chiyoda-ku, Tokyo, Japan.

PUBLISHED BY PACIFIC JOURNAL OF MATHEMATICS, A NON-PROFIT CORPORATION

The Supporting Institutions listed above contribute to the cost of publication of this Journal, but they are not owners of publishers and have no responsibility for its content or policies. 


\section{Pacific Journal of Mathematics}

\section{Vol. 27, No. $2 \quad$ February, 1968}

Leonard E. Baum and George Roger Sell, Growth transformations for

functions on manifolds ............................ 211

Henry Gilbert Bray, A note on CLT groups ................... 229

Paul Robert Chernoff, Richard Anthony Rasala and William Charles

Waterhouse, The Stone-Weierstrass theorem for valuable fields....... 233

Douglas Napier Clark, On matrices associated with generalized

interpolation problems ................................

Richard Brian Darst and Euline Irwin Green, On a Radon-Nikodym theorem for finitely additive set functions . ...................... 255

Carl Louis DeVito, A note on Eberlein's theorem..................... 261

P. H. Doyle, III and John Gilbert Hocking, Proving that wild cells exist . . . 265

Leslie C. Glaser, Uncountably many almost polyhedral wild $(k-2)$-cells in

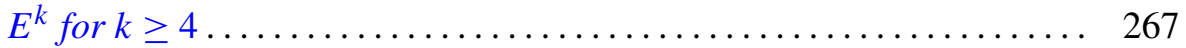

Samuel Irving Goldberg, Totally geodesic hypersurfaces of Kaehler

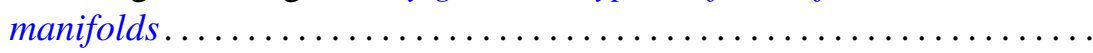

Donald Goldsmith, On the multiplicative properties of arithmetic functions .................................... 283

Jack D. Gray, Local analytic extensions of the resolvent ............ 305

Eugene Carlyle Johnsen, David Lewis Outcalt and Adil Mohamed Yaqub,

Commutativity theorems for nonassociative rings with a finite division ring homomorphic image ....................

André (Piotrowsky) De Korvin, Normal expectations in von Neumann

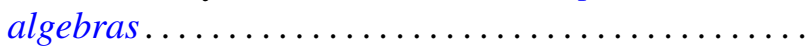

James Donald Kuelbs, A linear transformation theorem for analytic

Feynman integrals..........................

W. Kuich, Quasi-block-stochastic matrices ................... 353

Richard G. Levin, On commutative, nonpotent archimedean

semigroups ............................... 365

James R. McLaughlin, Functions represented by Rademacher series ... . . . 373

Calvin R. Putnam, Singular integrals and positive kernels............ 379

Harold G. Rutherford, II, Characterizing primes in some noncommutative

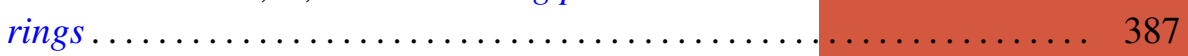

Benjamin L. Schwartz, On interchange graphs................... 393

Satish Shirali, On the Jordan structure of complex Banach *algebras . . . . . 397

Earl J. Taft, A counter-example to a fixed point conjecture............. 405

J. Roger Teller, On abelian pseudo lattice ordered groups ..... 